\title{
Exploring the QCD phase diagram at neutron star densities: the CBM experiment at FAIR
}

\section{Peter Senger* for the CBM collaboration}

GSI Helmholtzzentrum für Schwerionenforschung GmbH, Planckstr.1, 61291 Darmstadt, Germany, and Johann Wolfgang Goethe Universität, Frankfurt am Main, Germany

E-mail: p.senger@gsi.de

The Compressed Baryonic Matter (CBM) experiment will be one of the major scientific pillars of the future Facility for Antiproton and Ion Research (FAIR) in Darmstadt. The goal of the CBM research program is to explore the QCD phase diagram in the region of high baryon densities using high-energy nucleus-nucleus collisions. This includes the study of the equation-of-state of nuclear matter at neutron star core densities, and the search for the deconfinement and chiral phase transitions. The CBM detector is designed to measure rare diagnostic probes such as multi-strange hyperons, charmed particles and vector mesons decaying into lepton pairs with unprecedented precision and statistics. Most of these particles will be studied for the first time in the FAIR energy range. In order to achieve the required precision, the measurements will be performed at very high reaction rates of $100 \mathrm{kHz}$ to $10 \mathrm{MHz}$. This requires very fast and radiation-hard detectors, and a novel data read-out and analysis concept based on free streaming front-end electronics and a highperformance computing cluster for online event selection. The layout, the physics performance, and the status of the proposed CBM experimental facility will be discussed.

7th International Conference on Physics and Astrophysics of Quark Gluon Plasma

1-5 February, 2015

Kolkata, India

${ }^{*}$ Speaker. 


\section{Probing QCD Matter with Heavy-Ion Collisions}

High-energy heavy-ion collision experiments provide the possibility to create and investigate extreme states of strongly-interacting matter in the laboratory. Figure 1 illustrates the conjectured phases of nuclear matter and their boundaries in a diagram of temperature versus baryon chemical potential [1]. In experiments at LHC and top RHIC energies, matter is produced at very high energy densities with equal numbers of particles and antiparticles, i.e. at almost zero baryon chemical potential. After hadronization, the fireball finally freezes out chemically at a temperature of 155 - $165 \mathrm{MeV}$ [2]. This temperature coincides with the critical temperature predicted by Lattice QCD calculations for a chiral phase transition $[3,4]$ which is found to be a smooth crossover from partonic to hadronic matter [5]. Lattice QCD calculations for finite baryon chemical potential are still in their infancy, and are not yet able to make firm predictions on possible phase transitions at high net-baryon densities. On the other hand, model calculations predict structures in the QCD phase diagram at large baryon chemical potentials, like a critical endpoint followed by a first order phase transition [6]. Moreover, new phases are predicted, such as quarkyonic matter which can be considered as a Fermi gas of free quarks, with all thermal and Fermi surface excitations permanently confined [7]. The experimental discovery of these landmarks and regions in the QCD phase diagram would be a major breakthrough in our understanding of the properties of strongly interacting matter at extreme conditions, with fundamental consequences for our knowledge on the structure of neutron stars, chiral symmetry restoration, and the origin of hadron masses.

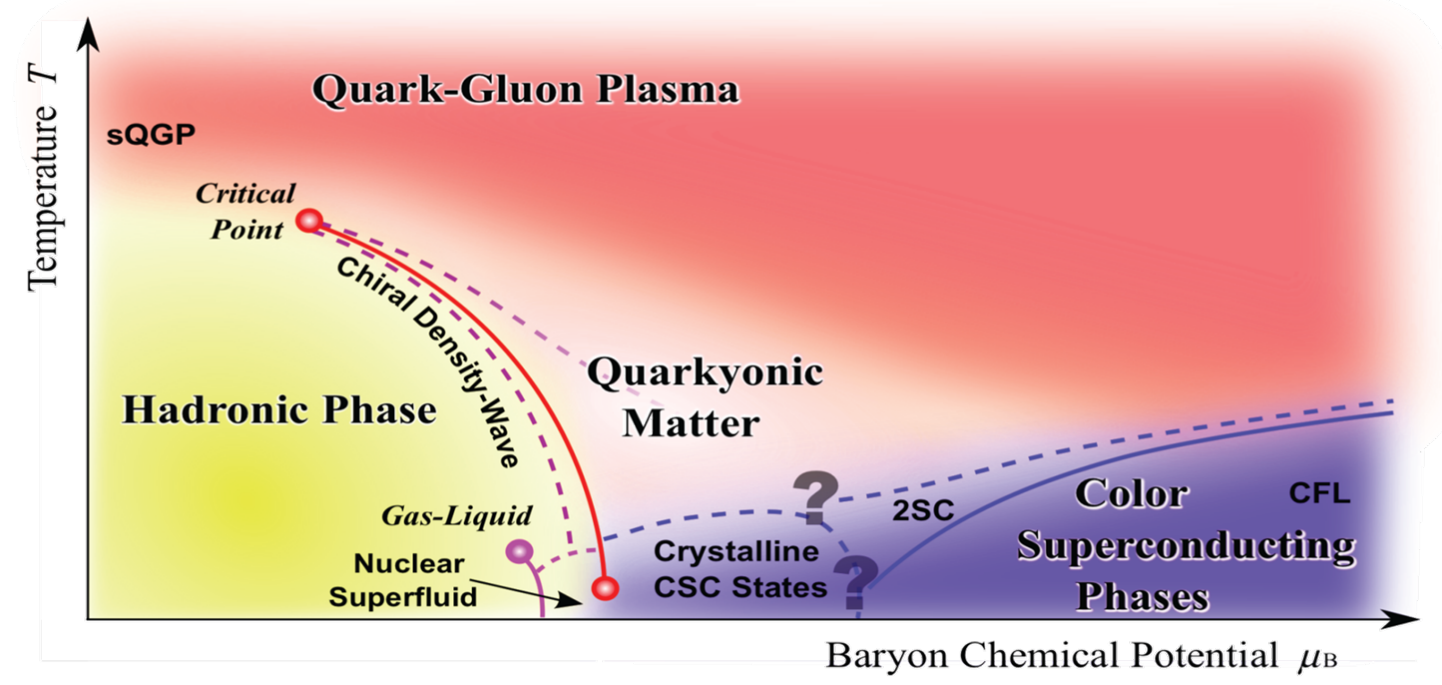

Figure 1: Sketch of the phase diagram for nuclear matter. Taken from [1].

\section{Experiments exploring high net-baryon densities}

Pioneering heavy-ion experiments have been performed at AGS in Brookhaven [8] and at low CERN-SPS beam energies [9] in order to explore the QCD phase diagram at large baryonchemical potentials. Due to the available detector technologies at that time these measurements 
were restricted to abundantly produced hadrons. At the CERN-SPS, the NA61/SHINE experiment continues to search for the first-order phase transition using light and medium size beams [10]. This detector setup is limited to reaction rates of about $80 \mathrm{~Hz}$. The STAR collaboration at RHIC plans a second beam energy scan to improve the statistical significance of the data taken in the first series of measurements [11]. However, the reaction rates at RHIC drop down to a few Hz when decreasing the beam energies below $\sqrt{s_{N N}}=8 \mathrm{GeV}$. Neither STAR at RHIC nor NA61 at SPS will be able to run at beam energies of $10 \mathrm{~A} \mathrm{GeV}$ and less. At the Joint Institute for Nuclear Research (JINR) in Dubna, a fixed target experiment is being developed at the Nuclotron to study heavy-ion collisions at goldbeam energies up to about $4 \mathrm{~A} \mathrm{GeV}$. Moreover, the collider facility NICA together with a multipurpose detector is planned at JINR [12]. The luminosity limitations of these existing and future facilities constrain the research programs to the investigation of bulk observables, and prevent high precision measurements of rare diagnostic probes. In contrast, the Compressed Baryonic Matter (CBM) experiment at the Facility for Antiproton and Ion Research (FAIR) is designed to run at extremely high interaction rates (up to $10 \mathrm{MHz}$ ). This feature is the key requirement for the measurement of both bulk and rare probes with unprecedented precision. The rate capabilities of existing and planned heavy-ion experiments are plotted in fig. 2 as function of beam energy. The combination of high-intensity beams with a high-rate detector system and sufficient beam time provides worldwide unique conditions for the study of nuclear matter at neutron star core densities. In the FAIR Modularized Start Version only the SIS100 ring will be available. In order to close the gap between SIS100 and low RHIC and SPS energies, SIS300 will be required. In addition, SIS300 will be used as a stretcher ring offering the opportunity to run several experimental programs in parallel.

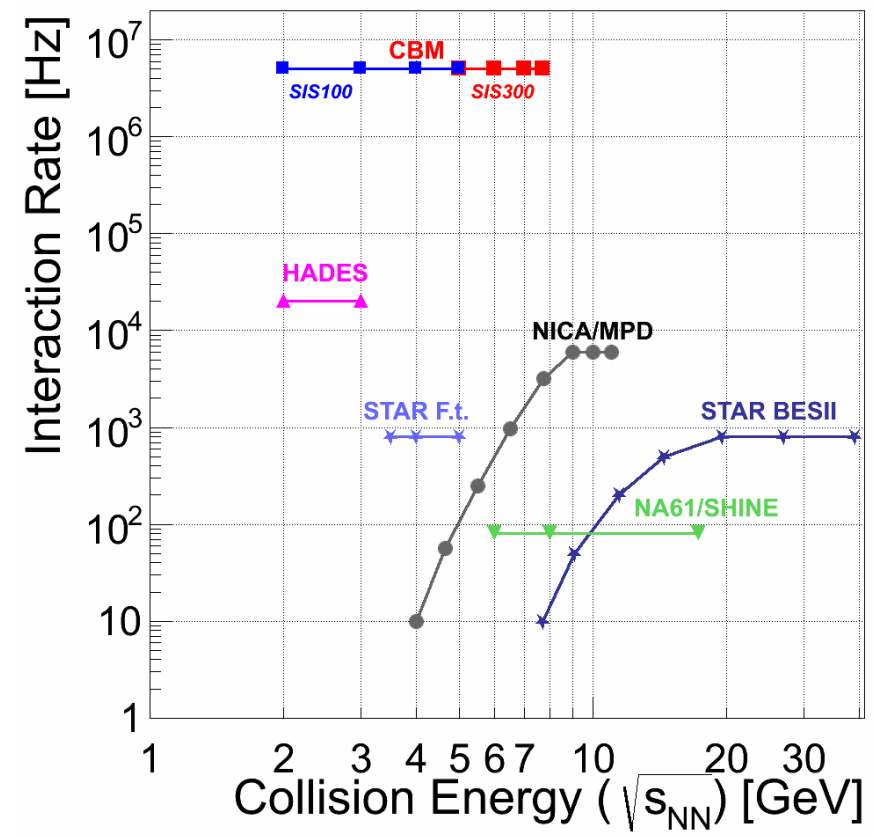

Figure 2: Interaction rates achieved by existing and planned heavy-ion experiments as function of beam energy. 


\section{The CBM Physics Program at SIS100}

FAIR will provide heavy-ion beam energies from 2 - 11 (14) A GeV for $\mathrm{Q}=0.4 \mathrm{~A}(0.5 \mathrm{~A})$ nuclei with the SIS100 synchrotron, and 11 - 35 (45) A GeV with the SIS300 synchrotron. Already in central $\mathrm{Au}+\mathrm{Au}$ collisions at $5 \mathrm{~A} \mathrm{GeV}$ the nuclear fireball will be compressed - according to transport model and hydro calculations - to more than 6 times saturation density $\rho_{0}$, and at $10 \mathrm{~A}$ $\mathrm{GeV}$ even a density above $8 \rho_{0}$ is reached as illustrated in figure 3 [13]. At such densities, the nucleons will start to melt and to dissolve into their constituents. The calculations predict that the dense fireball spends a relatively long time within the phase coexistence region or even beyond, as indicated in figure 3 by the dashed lines. Further indication, that a phase transition might occur at
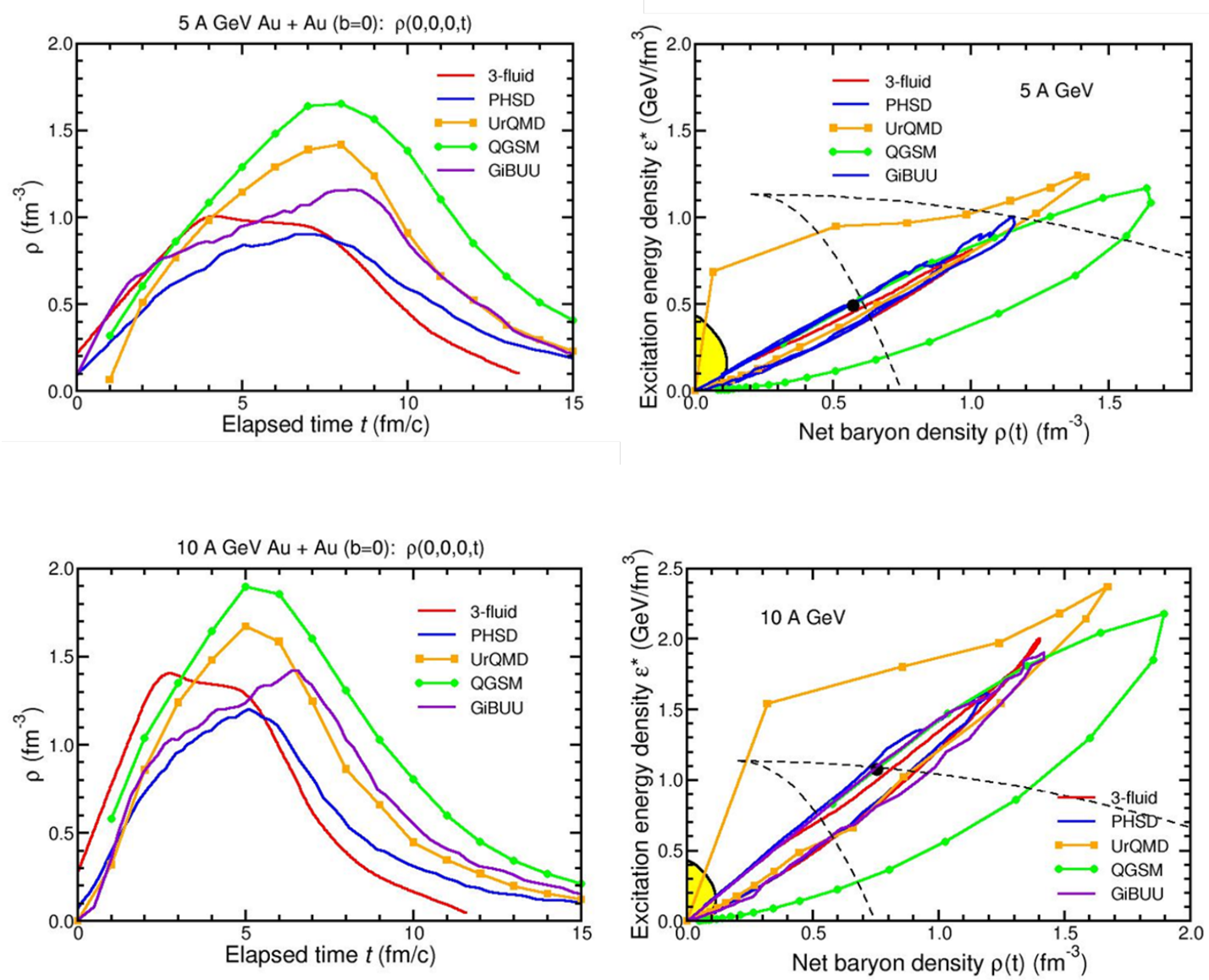

Figure 3: Results of different transport model calculations and of a 3-fluid hydrodynamics code for central $\mathrm{Au}+\mathrm{Au}$ collision at $5 \mathrm{~A} \mathrm{GeV}$ (upper panel) and at $10 \mathrm{~A} \mathrm{GeV}$ (lower panel). Left: Evolution of the central net baryon density $\rho(t)$ as function of elapsed time. Right: Excitation energy density as function of net baryon density. The dashed lines sketch the region of phase coexistence [13].

densities reached at SIS100 beam energies, comes from a non-local 3-flavor Nambu Jona-Lasinio model calculation of a neutron star, which predicts the development of a mixed phase of hadrons and quarks above densities of about $5 \rho_{0}$, and the transition to pure quark matter above $8 \rho_{0}$. This calculation ia able to reproduce a 2 solar mass neutron star [14]. In conclusion, the beam energies 
available at SIS100 appear to be especially well suited for generating signals of the phase transition, and, therefore, offer the opportunity to address fundamental scientific questions:

- What is the equation of state of nuclear matter at neutron star densities, and what are the relevant degrees of freedom at these densities? Is there a phase transition from hadronic to quark-gluon matter, or a region of phase coexistence? Do exotic QCD phases like quarkyonic matter exist?

- To what extent are the properties of hadrons modified in dense baryonic matter? Are we able to find indications of chiral symmetry restoration?

- How far can we extend the chart of nuclei towards the third (strange) dimension by producing single and double hypernuclei? Does strange matter exist in the form of heavy multi-strange objects?

- What is the production mechanism of charm quarks at threshold beam energies, how does open and hidden charm propagate in cold and in hot nuclear matter?

The experimental challenge is to identify diagnostic probes of the dense phase of the fireball which is transiently formed in the collision. In experiments performed so far at AGS, at low SPS energies, or at very low RHIC energies, mainly such particles have been measured which are created at freeze-out where the density already has dropped below saturation density. The focus of the CBM experiment at FAIR is to study messengers from the dense fireball such as multiple strange hyperons, lepton pairs, and hadrons containing charm quarks in order to find answers to the questions raised above. A survey of the theoretical concepts and the experimental programs devoted to the exploration of the QCD phase diagram with focus on high baryon densities is given in the CBM Physics Book [15]. The CBM research program at SIS100 includes the observables as discussed in the following.

\subsection{Strangeness}

At top SPS energies it was found that the production of hyperons is enhanced in heavy ion collisions, i.e. the multiplicity of hyperons per participating nucleon increases with the mass of the collision system [16]. The enhancement increases with the number of strange quarks in the hyperon. According to transport models, multi-strange (anti-)hyperons are produced in sequential collisions involving kaons and $\Lambda \mathrm{s}$, and, therefore, are sensitive to the density in the fireball. This sensitivity is expected to increase towards lower beam energies close to or even below the production threshold. SIS100 beam energies will be ideally suited to perform such experiments which require a systematic measurement of multi-(anti-)strange hyperons at different energies and for different colliding nuclei. Up to date, only about $300 \Xi^{-}$hyperons have been measured in $\mathrm{Au}+\mathrm{Au}$ collisions at $6 \mathrm{~A} \mathrm{GeV} \mathrm{[17].} \mathrm{The} \mathrm{excitation} \mathrm{functions} \mathrm{of} \mathrm{multi-strange} \mathrm{hyperons}\left(\Xi^{-}(d s s)\right.$ and $\left.\Omega^{-}(s s s)\right)$ and anti-hyperons $\left(\Xi^{+}(\bar{d} \bar{s} \bar{s})\right.$ and $\left.\Omega^{+}(\bar{s} \bar{s} \bar{s})\right)$ in A+A collisions with different A values at SIS100 beam energies are very promising observables which will shed light on the compressibility of nuclear matter at neutron star core densities. 


\subsection{Lepton pairs}

Lepton pairs will be measured over a wide range of invariant masses covering low-mass vector mesons including their Dalitz decays and charmonium. The precise measurement of lepton pairs at low invariant masses will allow to analyze modifications of vector meson properties in dense baryonic matter. This observable is expected to be sensitive to chiral symmetry restoration [18]. The slope of the dilepton invariant mass distribution between 1 and $2 \mathrm{GeV} / \mathrm{c}^{2}$ directly reflects the average temperature of the fireball. The study of the energy dependence of this slope opens the unique possibility to measure the caloric curve which would be a signature for phase coexistence [19]. This measurement would also provide indications for the onset of deconfinement and the location of the critical endpoint. The flow of lepton pairs as function of their invariant mass would allow to disentangle radiation from the early partonic phase from the late hadronic phase. No dilepton data are available at FAIR energies.

\subsection{Collective flow, correlations and fluctuations}

The collective flow of hadrons is driven by the pressure created in the early fireball. For example, the observed difference between the elliptic flow of particles and their antiparticles has been explained by mean-field potential effects in both the partonic and the hadronic phase [20]. A direct proof for a first order phase transition would be the discovery of phase coexistence by observing an enhanced production of composite particles or multi-particle correlations caused by the spinodal amplification of density fluctuations [21]. Higher moments of the net-baryon and the net-charge multiplicity distributions, which are related to the thermodynamical susceptibilities, have been measured by the STAR collaboration in order to search for the QCD critical point [22]. Most of these observables will be measured for the first time at SIS100 energies. Up to now, only the proton flow excitation function has been measured at the AGS [8], and was used to extract the compressibility of nuclear matter [23].

\subsection{Hypernuclei and hypermatter}

Theoretical models predict that single and double hypernuclei, and heavy multi-strange shortlived objects are produced via coalescence in heavy-ion collisions with the maximum yield in the region of SIS100 energies [24, 25]. The discovery and investigation of new hypernuclei and of hypermatter will shed light on the hyperon-nucleon and hyperon-hyperon interactions which are essential ingredients for the nuclear equation-of-state at high densities and low temperatures [26].

\subsection{Charm}

Charm-anticharm quark pairs are produced in primary hard scattering processes and then propagate through the nuclear or partonic medium as it is produced in high-energy heavy-ion collisions. Depending on their interaction with the medium, the charm and anticharm quarks hadronize into $D$ mesons, charmed baryons, or charmonium. The suppression of charmonium due to color screening of the heavy quark potential in the deconfined phase is the first predicted signature for quark-gluon plasma formation [27]. Charmonium suppression was first observed in central $\mathrm{Pb}+\mathrm{Pb}$ collisions at $158 \mathrm{~A} \mathrm{GeV} \mathrm{[28],} \mathrm{and} \mathrm{then} \mathrm{also} \mathrm{found} \mathrm{in} \mathrm{experiments} \mathrm{at} \mathrm{RHIC} \mathrm{[29]} \mathrm{and} \mathrm{LHC} \mathrm{[30].} \mathrm{At} \mathrm{SIS100}$ energies, the charm production mechanism will be studied for the first time at beam energies close 
to production threshold. Moreover, exploratory experiments of charm production in collisions of medium size nuclei will be possible. In addition, the propagation of open and hidden charm in cold nuclear matter will be systematically investigated in proton-nucleus collisions. Measurements of (open and hidden) charm in collisions of heavy nuclei can only be performed at SIS300 energies.

\section{The Compressed Baryonic Matter (CBM) Experiment}

The CBM detector is designed as a multi-purpose device which will be able to measure hadrons, electrons and muons in heavy-ion collisions over the full SIS100/SIS300 beam energy range. Therefore, no major adjustments have to be made to optimize the experiment for SIS100 beams, only some of the detectors and the DAQ system will be realized in phases (see below). In order to extract the dilepton signals, the physical and combinatorial background of lepton pairs has to be precisely determined, which is notoriously difficult. Measuring both electrons and muons will dramatically reduce the systematical error of the data, because the background sources of electrons and muons are completely different. In order to perform high-precision multi-differential measurements of rare probes the experiment should run at event rates of $100 \mathrm{kHz}$ up to $10 \mathrm{MHz}$ for several months per year. Because of the complicated decay topology of particles like $\Omega$ hyperons or $D$ mesons, no simple trigger signal can be generated, so the events have to be reconstructed and selected online by fast algorithms running on a high-performance computing farm. Therefore, the data readout chain is based on a free streaming frontend electronics which delivers time-stamped signals from each detector channel without event correlation. The reconstruction algorithms are tuned to run at high speed on modern many-core CPU architectures.

The detector system features a fixed target geometry accepting polar emission angles between 2.5 and 25 degrees in order to cover midrapidity for symmetric collision systems at beam energies between 2 and about $40 \mathrm{~A} \mathrm{GeV}$. The setup comprises the following components:

- A large aperture superconducting dipole magnet,

- a Silicon Tracking System (STS) based on double-sided silicon microstrip sensors arranged in 8 stations inside the magnetic field,

- a Micro Vertex Detector (MVD) consisting of 4 layers of silicon monolithic active pixel sensors,

- a time-of flight wall (TOF) based on multigap resistive plate chambers with low-resistivity glass for high-rate operation (up to $25 \mathrm{kHz} / \mathrm{cm}^{2}$ with a time resolution of $50 \mathrm{ps}$ ),

- a Ring Imaging Cherenkov (RICH) detector for electron identification comprising a $\mathrm{CO}_{2}$ radiator, glass-mirrors, and a photon detector based on multianode photomultipliers,

- a Transition Radiation Detector (TRD) for the identification of energetic electrons,

- a forward hadron calorimeter (Projectile Spectator Detector) for event characterization,

- a Muon chamber (MuCh) system for muon identification consisting of 5 triple stations of highly granulated gaseous micro-pattern chambers detectors sandwiched by iron plates with a total thickness equivalent to 13 absorption lengths,

- a First-Level-Event-Selection (FLES) system for online event reconstruction and selection. 
The RICH detector and the Muon chamber (MuCh) system will be used alternatively. All detector systems are equipped with self-triggered read-out electronics. After data compression and conversion into optical signals, the data are delivered via about $1000 \mathrm{~m}$ long fibres to the FAIR high performance computing cluster ("Green-IT cube") where the First Level Event Selection (FLES) will be performed. At high rate operation, a data volume of about 1 TByte will be delivered to the FLES, and about 1 GByte will finally be recorded.

The development of the experimental components is well in progress. The Technical Design Reports on the Superconducting Dipole Magnet, on the Silicon Tracking System, on the Ring Imaging Cherenkov Detectors, on the Projectile Spectator Detector, on the Time-of-Flight detector, and on the Muon Chamber system have been approved by FAIR. The TDRs on Data Acquisition and First Level Event Selection, on the Micro-Vertex-Detector, and on the Transition Radiation Detector will be submitted in 2016. A recent review of the experiment preparation is documented in the CBM Progress Report 2014 [31].

Until the start of FAIR, the nuclear matter research program at GSI will be pursued with the HADES experiment at SIS18. At the SIS100 accelerator, the HADES detector can be used to perform di-electron and hadron reference measurements in collision systems with moderate particle multiplicities, such as proton-proton, proton-nucleus and nucleus-nucleus collisions with light nuclei. A sketch of the CBM and HADES experimental setups is shown in figure 4 .

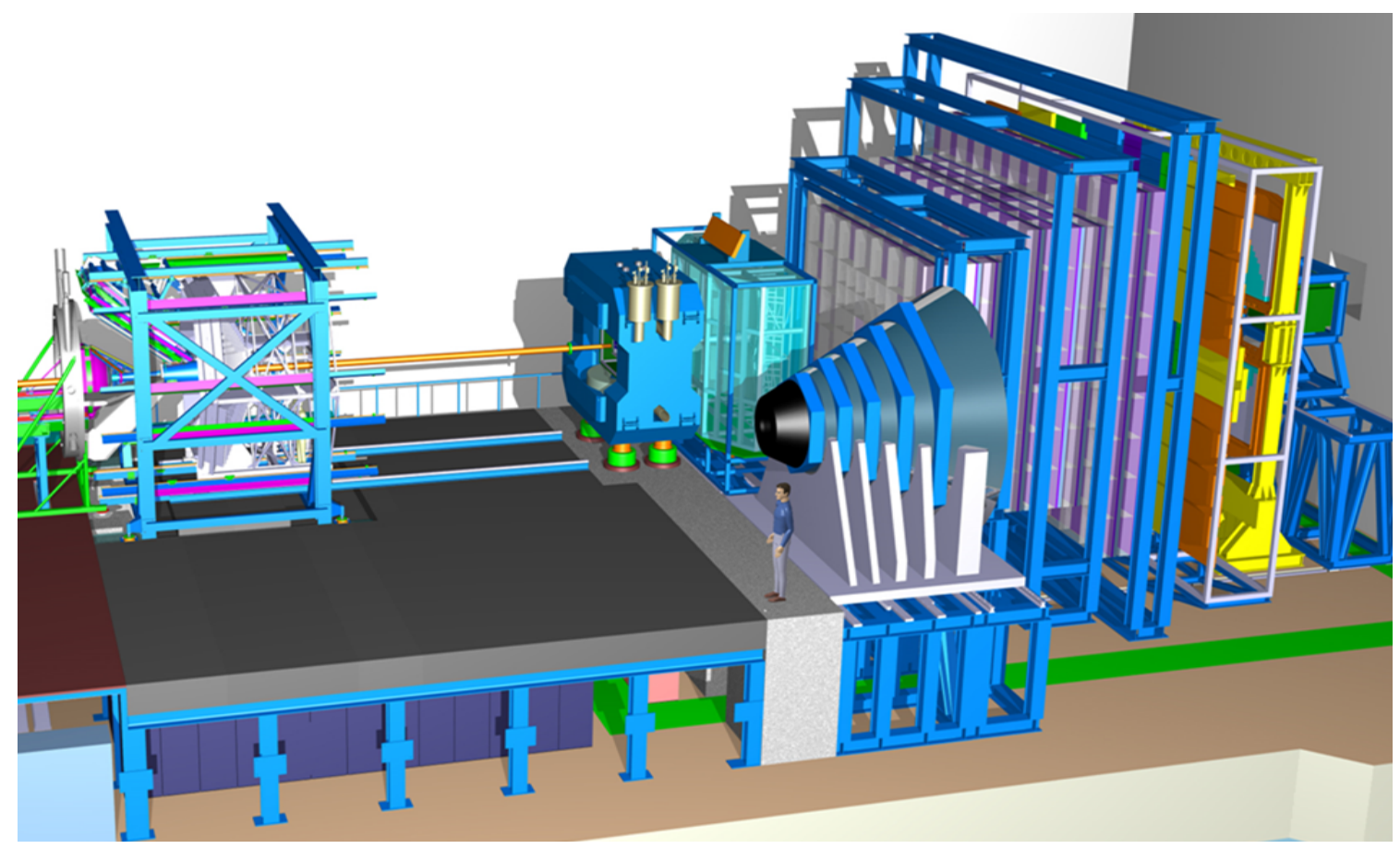

Figure 4: The HADES detector (left) and the CBM experimental setup (right) with the muon detection system in measuring position, and the RICH in parking position.

The optimization of the detector design has been carried out through extensive feasibility studies which are performed within a newly developed software framework ("FAIRroot"). The simulations involve event generators such as UrQMD and HSD, transport codes GEANT3/4 and FLUKA, 
and take into account realistic detector geometries, materials and response. The track reconstruction efficiency is better than 95\%, the momentum resolution is between 1 and $1.5 \%$ for momenta between 0.5 and $10 \mathrm{GeV} / \mathrm{c}$. Track reconstruction, searching for secondary vertices, particle identification using time-of-flight information, and calculation of invariant masses is performed by a high-speed algorithm running on many-core CPUs. The First Level Event Selection (FLES) package is vectorized, parallelized and scalable, more than 1600 events/sec can be processed by a single computing node with 80 cores. The algorithm provides invariant mass spectra of strange particles and resonances like $K_{s}^{0}, K^{*-}, K^{*+}, \phi, \Lambda, \bar{\Lambda}, \Xi^{-}, \Xi^{+}, \Omega^{-}, \Omega^{-}, \Sigma^{*-}, \Sigma^{*+}, \bar{\Sigma}^{*-}, \bar{\Sigma}^{*+}, \Xi^{* 0}, \bar{\Xi}^{* 0}$, and others. A selection of simulation results is presented in figure 5 , the yields are calculated for 1 million central $\mathrm{Au}+\mathrm{Au}$ collisions corresponding to a measuring time of several seconds. The CBM capabilities for dilepton measurements in central $\mathrm{Au}+\mathrm{Au}$ collisions at $8 \mathrm{~A} \mathrm{GeV}$ are demonstrated by the invariant mass spectra shown in figure 6 for low-mass electron-positron pairs (left panel) and for dimuon pairs (right panel). The simulations use the UrQMD code for background generation, and the HSD code for the yields of the signals which are imbedded in UrQMD events. The simulation and reconstruction of the electrons takes into account the information from the STS, the RICH, 4 TRD layers and the TOF detector. The muon simulations use information from the STS, the MuCh and the TOF detector.
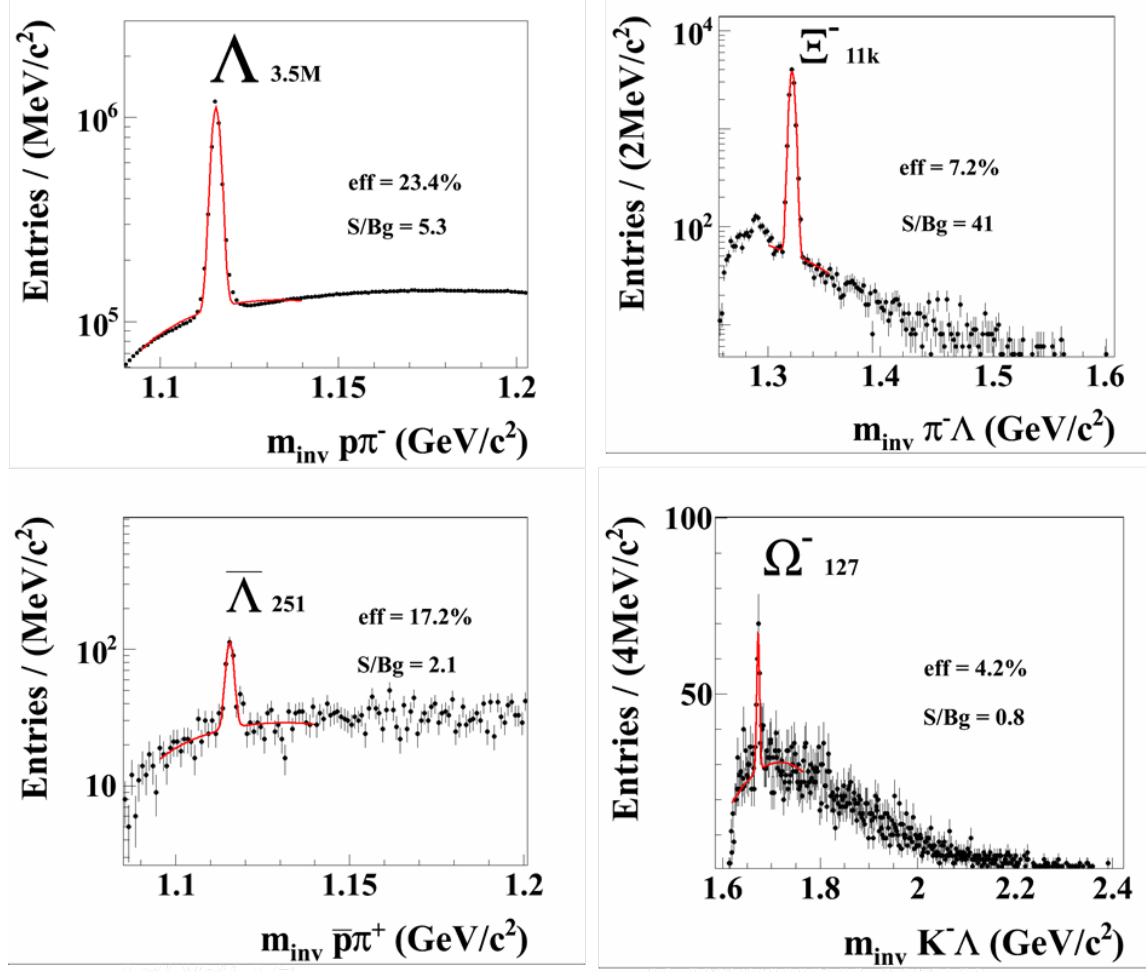

Figure 5: Invariant mass spectra of $p \pi^{-}$(upper left), $\pi^{-} \Lambda$ (upper right), $\bar{p} \pi^{+}$(lower left) and $K^{-} \Lambda$ (lower right) simulated and reconstructed for central $\mathrm{Au}+\mathrm{Au}$ collision at a beam energy of $8 \mathrm{~A} \mathrm{GeV}$ using the information from the STS and the TOF detector. The statistics corresponds to 1 million measured events. 

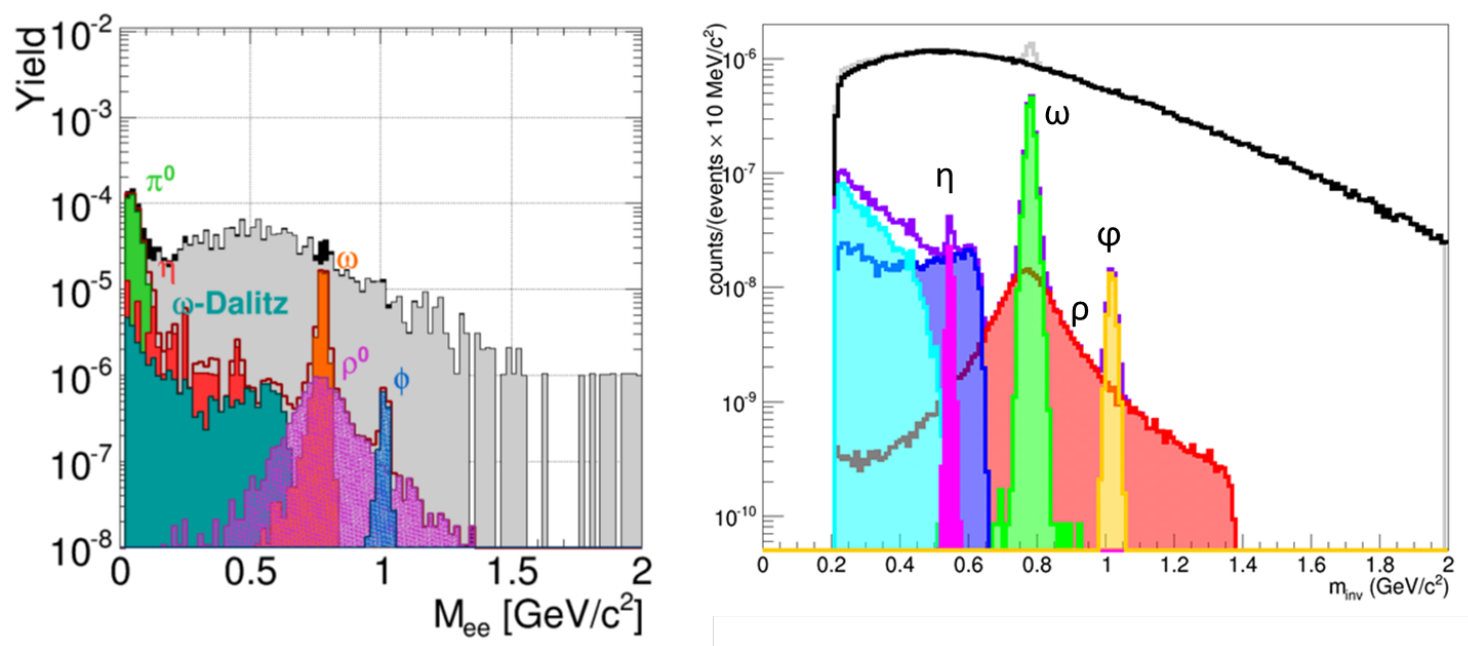

Figure 6: Invariant mass spectra of electron-positron pairs (left) and dimuon pairs (right) simulated for central $\mathrm{Au}+\mathrm{Au}$ collisions at $8 \mathrm{~A} \mathrm{GeV}$ (left). The dielectron spectrum corresponds to $2.5 \times 10^{5}$ events with a cut on $p_{t}>0.2 \mathrm{GeV} / \mathrm{c}$ for single electrons. The dimuon spectrum corresponds to $2.5 \times 10^{9}$ events. Different signal sources are shown with different colors: $\pi^{0}-, \eta$-, and $\omega$-Dalitz decays, $\rho$-, $\omega$-, and $\phi$-meson.

\section{Summary}

The Compressed Baryonic Matter (CBM) experiment will be one of the major scientific pillars of the future Facility for Antiproton and Ion Research (FAIR) in Darmstadt. The goal of the CBM research program is to explore the QCD phase diagram in the region of high baryon densities using high-energy nucleus-nucleus collisions. This includes the study of the equation-of-state of nuclear matter at neutron star core densities, and the search for the deconfinement and chiral phase transitions. The CBM detector is designed to measure rare diagnostic probes such as multi-strange hyperons, charmed particles and vector mesons decaying into lepton pairs with unprecedented precision and statistics. Most of these particles will be studied for the first time in the FAIR energy range. In order to achieve the required precision, the measurements will be performed at reaction rates between $100 \mathrm{kHz}$ and $10 \mathrm{MHz}$. This requires very fast and radiation hard detectors, and a novel data read-out and analysis concept based on free streaming front-end electronics and a highperformance computing cluster for online event selection. The use of the most modern detector and computer technology is the prerequisite for a large discovery potential of heavy-ion collision experiments at FAIR energies.

\section{Acknowledgments}

The design and development of the CBM experiment is performed by the CBM Collaboration which actually consists of more than 500 persons from 57 institutions and 12 countries. The CBM project is supported by the German Ministry of Education and Research, the Helmholtz Association, the EU FP7 Hadronphysics3 programme, and national funds of the CBM member institutions. 


\section{References}

[1] K. Fukushima and T. Hatsuda: Rept. Prog. Phys. 74 (2011) 014001

[2] F. Becattini et al., Phys. Rev. Lett. 111 (2013) 082302

[3] S. Borsanyi et al., JHEP 1009 (2010) 073

[4] A. Basavov et al.: Phys. Rev. D85 (2012) 054503

[5] Y. Aoki et al., Nature 443 (2006) 675

[6] J. Luecker et al., arXiv:1308.4509v1 [hep-ph]

[7] L. McLerran and R. D. Pisarski, Nucl. Phys. A796, 83-100 (2007),

[8] C. Pinkenburg et al., Phys. Rev. Lett. 83 (1999) 1295

[9] C. Alt et al., Phys. Rev. C77 (2008)024903

[10] A. Laszlo, Pos(CPOD07)054 (2007)

[11] https://drupal.star.bnl.gov/STAR/starnotes/public/sn0598

[12] http://nica.jinr.ru/

[13] I.C. Arsene et al.: Phys. Rev. C75 (2007) 24902

[14] M. Orsaria et al., Phys. Rev. C89 (2014)015806

[15] The CBM Physics Book, B. Friman et al.(eds): Series: Lecture Notes in Physics, Vol. 814 (2011)

[16] F. Antinori et al., Nucl. Phys. A661 (1999) 130c

[17] P. Chung et al., Phys. Rev. Lett. 91 (2003) 202301

[18] P. Hohler and R. Rapp, Phys. Lett. B731 (2014) 103-109

[19] R. Rapp and H. van Hees, arXiv:1411.4612

[20] J. Xu et al., Phys. Rev. Lett. 112 (2014) 012301

[21] J. Steinheimer and J. Randrup, Phys. Rev. Lett. 109 (2012) 212301

[22] L. Adamczyk et al., Phys. Rev. Lett. 113 (2014) 092301

[23] P. Danielewicz et al., Science 298 (2002) 1592

[24] A. Andronic et al., Phys. Lett. B697 (2011) 203

[25] H. Stoecker et al., Nucl. Phys. A827 (2009)

[26] A.S. Botvina et al., 1412.6665v1 [nucl-th]

[27] T. Matsui and H. Satz, Phys. Lett. B178 (1986) 416.

[28] M. Abreu et al., Phys. Lett. B410 (1997) 337.

[29] A. Adare et al., Phys. Rev. C84 (2011) 054912

[30] B. Abelev et al., Phys. Rev. Lett. 109 (2012) 072301

[31] V. Friese and C. Sturm (eds), ISBN 978-3-9815227-2-3. https://www-alt.gsi.de/documents/DOC-2015-Apr-43-1.pdf 